\title{
Strates
}

STRATES Matériaux pour la recherche en sciences sociales

$7 \mid 1993$

Témoins du monde : Bulgarie, identités chinoises, explorer l'île de France

\section{Vivre à l'échelle des livres}

\section{Michèle Petit}

\section{(2) OpenEdition}

Journals

Édition électronique

URL : http://journals.openedition.org/strates/1180

DOI : $10.4000 /$ strates. 1180

ISSN : $1777-5442$

Éditeur

Laboratoire Ladyss

Édition imprimée

Date de publication : 30 juin 1993

ISSN : 0768-8067

Référence électronique

Michèle Petit, «Vivre à l'échelle des livres », Strates [En ligne], 7 | 1993, mis en ligne le 20 décembre 2005, consulté le 08 septembre 2020. URL : http://journals.openedition.org/strates/1180; DOI :

https://doi.org/10.4000/strates. 1180

Ce document a été généré automatiquement le 8 septembre 2020

Tous droits réservés 


\title{
Vivre à l'échelle des livres
}

\author{
Michèle Petit
}

1 Au fil du récit qui suit, d'une génération à la suivante court une ambition, une ligne de vie : «aller très loin ", s'ouvrir à de nouveaux horizons. Pourtant ces Bigoudens sont très peu sortis de leur Bretagne natale : le père et la fille ne semblent l'avoir quittée, en fait, qu'à l'occasion d'une guerre. Mais s'ils ont peu voyagé, leurs yeux et leurs pensées ont couru partout. Et si leur espace s'est agrandi, s'il s'est ouvert sur d'autres continents, d'autres siècles, c'est avec les livres pour seul équipage.

2 Léontine Cariou raconte le pays bigouden de l'entre-deux-guerres: la «fin des terroirs $»^{1} \mathrm{y}$ est lente, on s'y déplace à pied, on s'y éclaire longtemps à la lampe pigeon, on parle breton, la dentelle court entre les doigts à la veillée, le dimanche on danse, mais pour tasser la terre : «on était toujours pour de l'utilité ». Marins ou paysans, la plupart sont très pauvres, et les livres rares. Pays entre mer et terre, dont Julien Gracq écrit: «si clos, si cloîtré était leur monde de la terre ferme - à peine doué de consistance tellement il s'ouvrait de partout sur le large... $»^{2}$ Pays aussi entre Blancs et Rouges, où les curés prêchent contre «l'école du diable», et où les bateaux s'appellent tantôt «Grâce de Dieu », tantôt «Lumière d'Octobre " ${ }^{3}$.

3 C'est un couple ainsi bigarré, celui de ses parents, qu'évoque d'abord Léontine Cariou : elle va à la messe, lui lira le « Populaire». A l'origine de la passion de lire qui prendra le père et sa fille, il y a déjà la voix de la mère, leur lisant des vies de saints, cette voix dont on sait l'importance pour ouvrir le chemin qui mène à la lecture ${ }^{4}$. Puis les pages qu'elle écrit et envoie à son mari cantonnier, parti à la Grande Guerre ${ }^{5}$ : à force de rapprocher et de comparer les lettres énigmatiques que dessine sa femme, tel Champollion il arrive à percer leur mystère. Et il se jette, à son retour, sur les livres de lecture de ses enfants. Sa fille, posée sur un banc à l'école à deux ans et demi, à son tour observe et décrypte le tableau où les plus grands jouent avec l'alphabet.

Avide de tout savoir, elle deviendra institutrice. Elle appartient à ces générations de maîtres ${ }^{6}$ épris des Lumières qui ont espéré que le livre, et l'école, couvrant peu à peu le territoire, pourraient former les lecteurs, réformer la société, déjouer les inégalités sociales ou spatiales : aider à "ouvrir » ces campagnes où la distance physique qui séparait des livres redoublait la difficulté d'accès social et psychique à ces objets 
imprimés avec lesquels les ruraux entretenaient souvent un rapport ambivalent, fait de fascination et d'évitement ${ }^{7}$.

5 Beaucoup de ces instituteurs n'avaient-ils pas eux-mêmes reçu le monde en partage grâce aux livres? Et telle Léontine Cariou, fière d'être Bretonne, qui habite aussi le monde en citoyenne responsable et en poète, ne s'étaient-ils pas réclamés d'une identité ouverte?

6 L'accès aux différents «biens culturels » n'est ainsi pas seulement affaire de goût, de rêverie, de savoir, ou de distinction sociale, mais aussi d'ouverture sur des espaces plus ou moins larges. Il est des pratiques culturelles qui, plus que d'autres, rivent aux seules racines, contribuent à « boucler » celle ou celui qui s'y adonne dans sa tribu, à sa place : telle la lecture exclusive du journal local. Et il en est d'autres qui font vaciller les ancrages, dépaysent. Elles donnent une chance de redessiner sa carte mentale, d'y faire figurer non seulement son lieu d'appartenance, mais aussi des espaces de référence à partir desquels imaginer, penser, des échanges, des projets. Et elles ouvrent une possibilité de sortir de la loi du lieu, du déterminisme sociologique, de la reproduction à l'identique. De trouver un peu de jeu dans les places désignées.

7 Ce ne sont pas forcément les seuls biens culturels "nobles» qui ouvrent l'horizon: dans notre siècle où les campagnes se sont peu à peu désenclavées, des images découpées dans des almanachs, des cartes postales, des timbres ou des vignettes trouvées dans les tablettes de chocolat ont quelquefois ouvert les maisons rurales sur des espaces élargis : vedute modestes, elles ont permis de se perdre dans le lointain, dans des chemins, des villes, de se transporter ailleurs, comme ces fenêtres qu'à la veille du siècle des découvreurs les peintres commençaient à percer dans l'espace jusque là clos des scènes qu'ils représentaient.

Il y eut aussi la TSF, la «boîte rouge » que Léontine Cariou laisse à ses parents, cette radio dont Michel Cournot disait qu'elle nous inoculait le monde, qu'on n'en guérissait plus ${ }^{8}$. Puis la télévision : quand elle a offert un poste à ses parents, "ça leur a ouvert le monde ». Aujourd'hui, bien des émissions l'ont plutôt refermé, et le «village» y fait furieusement retour ${ }^{9}$, en ces temps où mondialisation et enracinement local se donnent la main.

9 Et il y eut donc aussi les journaux et les livres. La lecture a toujours plusieurs faces. D'un côté, elle peut permettre de contrôler à distance, apprendre à "se tenir » à sa place, par des gestes d'identification à des modèles largement diffusés. D'un autre côté, par excellence dans la fiction, elle génère de la singularité, et elle transporte ailleurs : tel le gitan de Cent ans de solitude apportant chaque année à Macondo les découvertes de ce lointain qui commençait au-delà des sierras, les livres épellent les autres lieux, permettent de voir loin, d'un bout de la terre à l'autre. Et ils contribuent quelquefois à fabriquer des transfuges, qui sautent au-dessus du groupe et prennent la route...

10 Aujourd'hui où, dans les campagnes et dans les villes, tant se sentent "enfermés hors du monde », pour parler comme Cavafy, et se replient sur des identités d'appartenance à leur paroisse, il peut être urgent d'en savoir plus sur « l'espace» dans lequel inscrit chacune de ces activités que l'on recouvre du terme indifférencié de "pratiques culturelles ».

11 Et rendre publiques la rigueur bienveillante et l'enthousiasme de Léontine Cariou, c'est un peu rendre hommage à la modernité de celles et de ceux qui, sans jamais renier leurs origines, se sont aussi voulus citoyens du monde, ouverts à tout ce qui venait. Et 
qui ont plongé dans les atlas et sont allés trouver au creux des livres le «soleil» des penseurs d'autres siècles, ou "la délicatesse, la soie et les fleurs japonaises » des écrivains lointains.

12 Les livres ne sont-ils pas eux-mêmes des fleurs japonaises, ces objets qui nous faisaient rêver quand nous étions enfants, de papier gris plié, qui posés sur l'eau ouvraient leur corolle, étiraient leurs pétales, prenaient mille couleurs, et nous emportaient...

\section{BIBLIOGRAPHIE}

Quelques références bibliographiques sur le pays bigouden:

BREKILIEN Yann, La vie quotidienne des paysans en Bretagne au XIX ${ }^{e}$ siècle, Paris, Hachette, 1972.

BURGUIERE André, Bretons de Plozévet, Paris, Flammarion, 1975.

FLATRES Pierre, La Bretagne, Paris, PUF, 1986.

HELIAS Pierre-Jakez, Le quêteur de mémoire, Paris, Plon/Terre humaine, 1990.

HELIAS Pierre-Jakez, Le cheval d'orgueil, Paris, Plon/Terre humaine, 1975.

HELIAS Pierre-Jakez, LE GALL Yves, QUEFFELEC Henri, Penn ar Bed - Finistère, Brest, Ed. de la Cité, 1977.

LAMBERT Yves, BREMAUD M-A, « Limerzel, quatre-vingt ans de catholicisme en Bretagne », Cahiers de l'observation du changement social, CNRS, 4, 1982.

LE LANNOU Maurice, La Bretagne et les Bretons, Paris, PUF, Que sais-je ?, 1978.

MATHIEU Nicole, Les transformations socio-économiques d'une commune rurale : Plozévet 1820-1920, Publications de l'AUDIR, Paris, Hachette, 1973.

MORIN Edgar, Campagne en France : la métamorphose de Plodémet, Paris, Fayard, 1967.

PHLIPPONNEAU Michel, Debout Bretagne, Saint-Brieuc, PUF de Bretagne, 1970.

PIRIOU Nicole et CLEAC'H, « La déprise agricole et la recomposition de l'espace littoral sudbigouden », in Géographie sociale, « Quelles campagnes pour demain ? », 12, Caen, CNRS URA 915, sept. 1992, pp. 215-226.

SAINCLIVIER Jacqueline, La Bretagne de 1939 à nos jours, Ed. Ouest-France Univers, 1989.

Revue Cap-Caval, Pont-l'Abbé.

SEGALEN Martine, Quinze générations de Bas-bretons. Mariage, parentèles et sociétés dans le pays bigouden sud, 1720-1980, Paris, PUF, 1985.

\section{NOTES}

1. Pour reprendre l'expression d'Eugen WEBER qui donne son titre à son ouvrage : La fin des terroirs, la modernisation de la France rurale, 1870-1914, Paris, Fayard, 1983. 
2. Cf. Carnets du grand chemin, Paris, José Corti, 1992, p. 134.

3. Op. cit., p. 133.

4. Voir par exemple François de SINGLY : «Le poids des grands lecteurs est deux fois plus important parmi ceux qui ont bénéficié d'une histoire comptée par leur mère chaque jour que parmi ceux qui n'en ont écouté aucune (ou rarement). » (Les jeunes et la lecture, Ministère de l'Education nationale et de la Culture, Dossiers Educations et formations, 24, janvier 1993, p. 102).

5. Notons que c'est aussi durant la guerre de quatorze que la lecture du journal s'est généralisée, car elle permettait de suivre les nouvelles du front.

6. Cf. à ce sujet l'ouvrage de Mona et Jacques OZOUF, 1992, La République des instituteurs, Paris, Gallimard/Seuil, qui porte sur la génération antérieure, mais où l'on retrouve souvent des représentations évoquées par notre interlocutrice.

7. Cf. Claude-Michèle GARDIEN, Raymonde LADEFROUX et Michèle PETIT, Lecteurs en campagnes, Editions de la Bibliothèque Publique d'Information du Centre Georges Pompidou (collection Etudes et recherches), à paraître en automne 1993.

8. Cf. « La force de l'absence ", in Le Nouvel Observateur, 6/4/1970 (à propos du Droit de rêver de Gaston BACHELARD, Paris, PUF).

9. Cf. Serge DANEY, 1991, Devant la recrudescence des vols de sacs à main, cinéma, télévision, information, Lyon, Aléas, « Le prime time du village », Libération, 11/3/1992 et « La colère du zappeur », Nouvel Observateur, 18/7/1991. Cf. aussi Régis DEBRAY,1992, Vie et mort de l'image, Paris, Gallimard, chapitre XI.

\section{AUTEUR}

\section{MICHÈLE PETIT}

Ingénieur au CNRS (STRATES). Anthropologue en pays de géographes, elle privilégie les méthodes qui prennent en compte l'expérience singulière des acteurs composant le social. Ses travaux actuels portent sur le rôle des pratiques culturelles dans la reformulation des identités. Elle est co-auteur de l'ouvrage Lecteurs en campagnes (Editions de la BPI, Centre Georges Pompidou, à paraître en automne 1993). 